\title{
Improving outcomes in patients with non-tuberculous mycobacterial disease: is there light at the end of the tunnel?
}

\author{
Keertan Dheda ${ }^{1,2}$ \\ Affiliations: ${ }^{1}$ Centre for Lung Infection and Immunity, Division of Pulmonology, Dept of Medicine and UCT \\ Lung Institute and South African MRC/UCT Centre for the Study of Antimicrobial Resistance, University of \\ Cape Town, Cape Town, South Africa. ${ }^{2}$ Faculty of Infectious and Tropical Diseases, Dept of Immunology and \\ Infection, London School of Hygiene and Tropical Medicine, London, UK.
}

Correspondence: Keertan Dheda, Centre for Lung Infection and Immunity Unit, Division of Pulmonology, Dept of Medicine, University of Cape Town, H46.41 Old Main Building, Groote Schuur Hospital, Observatory, 7925 South Africa. E-mail: keertan.dhedaduct.ac.za

@ERSpublications

Improving outcomes in patients with non-tuberculous mycobacterial diseases http://bit.ly/2ITj8O2

Cite this article as: Dheda K. Improving outcomes in patients with non-tuberculous mycobacterial disease: is there light at the end of the tunnel? Eur Respir J 2019; 54: 1901149 [https://doi.org/10.1183/ 13993003.01149-2019].

This issue of the European Respiratory Journal presents a series of research papers, reviews and editorials on the emerging problem of non-tuberculous mycobacterial (NTM) disease, the burden of which is increasing in many parts of the world [1]. Thus, NTM-related disease is a burgeoning public health problem even in areas where tuberculosis is endemic. Non-tuberculous mycobacteria are hardy environmental saprophytes and opportunistic pathogens that usually afflict those with structural lung disease and/or a compromised immune system due to old age or other reasons, including poorly characterised hereditary or acquired defects in innate or adaptive immunity [2]. Although there many causes of NTM pulmonary disease (NTM-PD), Mycobacterium avium complex (MAC), Mycobacterium kansasii and Mycobacterium abscessus (MAB) are generally the most commonly seen causative agents in many parts of the world [1].

There are several important questions regarding the optimal management of NTM disease. Will we be able to eventually transcend to a pan-oral regimen for NTM disease, and are newer anti-mycobacterial drugs like bedaquiline and delamanid, and repurposed ones like linezolid, useful in improving outcomes in patients with NTM disease? Bedaquiline is a particularly attractive option because it is now globally available and has been shown to substantially reduce mortality and improve treatment outcomes in patients with multidrug-resistant tuberculosis (MDR-TB) [3-5]. A paper in this issue of the European Respiratory Journal by ZweIJPFENNING et al. [6] explores this question. They investigated the use of bedaquiline for MAC-related pulmonary disease and highlight the challenge in managing patients with NTM disease, including dealing with attendant drug toxicity, and selecting a salvage regimen for treatment failures who often either refuse or are not candidates for lung resection. The report also highlights the potential danger of adding a single drug to a failing regimen with the development of acquired resistance to bedaquiline. Although this report describes a single case, the authors must be commended on conducting comprehensive longitudinal genomic susceptibility testing using next generation whole genome sequencing and using therapeutic drug monitoring to guide management. As outlined in several case reports (referenced in the report), many MAC isolates are sensitive to bedaquiline but, importantly, 
rifamycins, a critical component of any MAC regimen, cannot generally be combined with bedaquiline as it lowers exposure by $75 \%$ through induction of liver enzymes [7]. The safety of increased doses of bedaquiline remains to be clarified. There is also potential cross-resistance between bedaquiline and clofazimine $[8,9]$. A second issue highlighted by this report is the poor drug-specific responsiveness to treatment despite continued sensitivity of mycobacterial isolates to the same drugs. This is not an uncommon scenario seen when managing patients with mycobacterial disease in general, including those with drug-sensitive and drug-resistant tuberculosis. When seen, this could potentially be explained by a number of factors, including non-adherence, malabsorption and hetero-resistance, but is often likely to be due to poor antibiotic penetration into thick-walled cavities. We recently showed this to be an important consideration in patients with drug-resistant tuberculosis when intra-cavitary drug levels were measured [10]. This raises the question of whether adjunct inhaled antibiotics (inhaled antibiotics in addition to oral or parenteral ones) and therapeutic drug monitoring may improve outcomes when used to treat or guide NTM disease. Further research is now required to determine the optimal dose of anti-mycobacterial drugs required for satisfactory intra-cavitary penetration.

Although there are several case reports of bedaquiline failure and acquired resistance in the setting of MAC-related pulmonary disease, should we abandon this as a treatment option for MAC or other types of NTM disease? I think not. Thus far, the experience has been in a small number of cases and several factors could have contributed to the acquired resistance and failure, including poor cavity penetration, hetero-resistance, poor adherence, and reduced exposure of bedaquiline by concomitant rifamycin usage, etc. Controlled studies are now required, including with therapeutic drug monitoring, to determine the role of bedaquiline in the treatment of NTM disease. There are also limited data on the use of repurposed drugs such as linezolid for NTM disease. More recently, linezolid has been shown to reduce mortality and improve treatment-related outcomes in patients with MDR-TB, and is now part of a frontline pan-oral regimen for MDR-TB [11]. Although efficacy is region- and strain-specific, promising data are now emerging about the usefulness of oxazolidinones such as linezolid and tedizolid for NTM infections [12-14].

NTM-PD is often challenging to treat: sometimes the disease cannot be eradicated, and toxicity and compliance are major challenges. M. abscessus-related pulmonary disease (MAB-PD), and in particular MAB-PD due to $M$. abscessus subspecies abscessus, is particularly difficult to manage. Thoracic societies have provided recommendations for the treatment of MAB-PD, which generally includes one or more parenteral drugs (amikacin and/or imipenem), a macrolide, and other antibiotics [15, 16]. The lack of standardised high sensitivity genomic readouts, and availability and standardisation of phenotypic susceptibility testing assays and breakpoints, means that treatment is often empirical. However, there are several unanswered questions regarding the optimal treatment of NTM-PD in general, and MAB-PD in particular. For example, what is the optimal duration of treatment for MAB-PD, and what is the minimum number of likely effective drugs required for optimal treatment outcomes? Furthermore, which specific individual drugs improve treatment-related outcomes? These are critical questions because the decision to treat MAB-PD must be carefully weighed up against likely efficacy of a regimen, potential toxicity (which may be considerable) and, invariably, the need for a tunnelled intravenous catheter with its attended risks of infection and other complications, not to mention cost to the patient and the healthcare system. A paper by KWAK et al. [17] in this issue of the European Respiratory Journal attempts to address some of these important questions. They conducted an individual patient data meta-analysis using studies that reported treatment outcomes for MAB-PD that included 303 patients. Their key finding was that the use of imipenem was associated with significantly better treatment success in MAB-PD (as a collective and without subspecies identification). In patients with subspecies abscessus, in particular, they found that azithromycin, amikacin and imipenem were associated with a significantly higher likelihood of treatment success and better outcomes. There are several limitations of this study, many of which the authors acknowledge, including: not all published outcome-based studies were included in their analysis; datasets were limited in some cases; there was a suggestion of publication bias; the incorporated studies were often retrospective in design; and there was failure to correct for mycobacterial burden and pre-treatment disease severity. However, the key strength, as the authors point out, was that this was the first individual patient data meta-analysis attempting to interrogate specific drugs associated with a favourable outcome in MAB-PD.

What are the implications of these findings for clinical practice? First, notwithstanding the limitations, the data suggest that imipenem should form the backbone of any MAB-PD treatment regimen, with which other antibiotics can be combined and the resulting regimen modified based on the susceptibility testing readouts. For subspecies abscessus the same data suggest that a combination of azithromycin, imipenem and/or amikacin should likely form the backbone of a regimen with the highest likelihood of success. Thus, in MAB-PD due to subspecies abscessus, azithromycin was associated with better outcomes than clarithromycin. It is interesting that, unlike with M. tuberculosis, the addition of a beta-lactamase inhibitor 
like clavulanate or tazobactam does not improve the efficacy of the carbapenem (as M. abcessus produces a clavulanate-insensitive broad-spectrum $\beta$-lactamase) [18].

The second major finding of the study was that outcomes were poor, as confirmed by two other meta-analyses [19, 20]. Indeed, treatment success rates were only 33\% for MAB-PD subspecies abscessus [17] and similar to that of extensively drug-resistant tuberculosis in the pre-bedaquiline era [21]. This also raises the question of how successful outcomes are defined for NTM disease? The authors defined treatment success as culture conversion after $\geqslant 12$ months of treatment or sustained culture conversion without relapse until the end of treatment. More work is required to delineate robust outcome definitions for NTM disease and the positive and negative predictive value of 12-month culture conversion for each major causative agent of NTM disease.

Where to from here? There is a need to combine data and use an individual patient meta-analysis approach to redefine a hierarchy of effective drugs for the optimal treatment of each type of NTM disease. This approach was recently effectively employed to guide, inform and dramatically improve MDR-TB outcomes [22]. In tandem, experimental anti-mycobacterial drugs and other interventions [23], including host-directed therapies to augment host immunity [24], therapeutic drug monitoring and adjunct inhaled antibiotics, need to be evaluated to improve the outcomes of this burgeoning disease. NTM is a tough nut to crack but through using a combination of these approaches it is likely that, in the future, we will transcend to effective and shortened pan-oral regimens for different types of NTM disease. Thus, it's not all dark and bleak. There is light at the end of the NTM tunnel!

\section{References}

1 Brode SK, Daley CL, Marras TK. The epidemiologic relationship between tuberculosis and non-tuberculous mycobacterial disease: a systematic review. Int J Tuberc Lung Dis 2014; 18: 1370-1377.

2 Namkoong H, Hasegawa N, Betsuyaku T. Susceptibility genes for nontuberculous mycobacterial disease. Nihon Rinsho Meneki Gakkai Kaishi 2017; 40: 60-67.

3 Olayanju O, Limberis J, Esmail A, et al. Long-term bedaquiline-related treatment outcomes in patients with extensively drug-resistant tuberculosis from South Africa. Eur Respir J 2018; 51: 1800544.

4 Diacon AH, Pym A, Grobusch MP, et al. Multidrug-resistant tuberculosis and culture conversion with bedaquiline. $N$ Engl J Med 2014; 371: 723-732.

5 Schnippel K, Ndjeka N, Maartens G, et al. Effect of bedaquiline on mortality in South African patients with drug-resistant tuberculosis: a retrospective cohort study. Lancet Respir Med 2018; 6: 699-706.

6 Zweijpfenning SMH, Schildkraut JA, Coolen JPM, et al. Failure with acquired resistance of an optimised bedaquiline-based treatment regimen for pulmonary Mycobacterium avium complex disease. Eur Respir J 2019; 54: 1900118.

7 Svensson EM, Murray S, Karlsson MO, et al. Rifampicin and rifapentine significantly reduce concentrations of bedaquiline, a new anti-TB drug. J Antimicrob Chemother 2015; 70: 1106-1114.

8 Hartkoorn RC, Uplekar S, Cole ST. Cross-resistance between clofazimine and bedaquiline through upregulation of MmpL5 in Mycobacterium tuberculosis. Antimicrob Agents Chemother 2014; 58: 2979-2981.

9 Somoskovi A, Bruderer V, Homke R, et al. A mutation associated with clofazimine and bedaquiline cross-resistance in MDR-TB following bedaquiline treatment. Eur Respir J 2015; 45: 554-557.

10 Dheda K, Lenders L, Magombedze G, et al. Drug penetration gradients associated with acquired drug resistance in tuberculosis patients. Am J Respir Crit Care Med 2018; 198: 1208-1219.

11 WHO Consolidated Guidelines. Drug-resistant tuberculosis treatment. Geneva, World Health Organization, 2019. Report No.: WHO/CDS/TB/2019.7.

12 Brown-Elliott BA, Wallace RJ Jr. In vitro susceptibility testing of tedizolid against nontuberculous mycobacteria. J Clin Microbiol 2017; 55: 1747-1754.

13 Compain F, Soroka D, Heym B, et al. In vitro activity of tedizolid against the Mycobacterium abscessus complex. Diagn Microbiol Infect Dis 2018; 90: 186-189.

14 Deshpande D, Srivastava S, Pasipanodya JG, et al. Linezolid as treatment for pulmonary Mycobacterium avium disease. J Antimicrob Chemother 2017; 72: Suppl. 2, i24-i29.

15 Griffith DE, Aksamit T, Brown-Elliott BA, et al. An official ATS/IDSA statement: diagnosis, treatment, and prevention of nontuberculous mycobacterial diseases. Am J Respir Crit Care Med 2007; 175: 367-416.

16 Haworth CS, Banks J, Capstick T, et al. British Thoracic Society guidelines for the management of non-tuberculous mycobacterial pulmonary disease (NTM-PD). Thorax 2017; 72: Suppl. 2, ii1-ii64.

17 Kwak N, Dalcolmo MP, Daley CL, et al. Mycobacterium abscessus pulmonary disease: individual patient data meta-analysis. Eur Respir J 2019; 54: 1801991.

18 Soroka D, Dubee V, Soulier-Escrihuela O, et al. Characterization of broad-spectrum Mycobacterium abscessus class A beta-lactamase. J Antimicrob Chemother 2014; 69: 691-696.

19 Diel R, Ringshausen F, Richter E, et al. Microbiological and clinical outcomes of treating non-mycobacterium avium complex nontuberculous mycobacterial pulmonary disease: a systematic review and meta-analysis. Chest 2017; 152: 120-142.

20 Pasipanodya JG, Ogbonna D, Ferro BE, et al. Systematic review and meta-analyses of the effect of chemotherapy on pulmonary Mycobacterium abscessus outcomes and disease recurrence. Antimicrob Agents Chemother 2017; 61: e01206-17.

21 Pietersen E, Ignatius E, Streicher EM, et al. Long-term outcomes of patients with extensively drug-resistant tuberculosis in South Africa: a cohort study. Lancet 2014; 383: 1230-1239. 
22 Collaborative Group for the Meta-Analysis of Individual Patient Data in MDR-TB treatment 2017, Ahmad N, Ahuja SD, et al. Treatment correlates of successful outcomes in pulmonary multidrug-resistant tuberculosis: an individual patient data meta-analysis. Lancet 2018; 392: 821-834.

23 Felicetti T, Machado D, Cannalire R, et al. Modifications on C6 and C7 positions of 3-phenylquinolone efflux pump inhibitors led to potent and safe antimycobacterial treatment adjuvants. ACS Infect Dis 2019; 5: 982-1000.

24 Stek C, Allwood B, Walker NF, et al. The immune mechanisms of lung parenchymal damage in tuberculosis and the role of host-directed therapy. Front Microbiol 2018; 9: 2603. 